\title{
Experimental Assessment of a Programmable VCSEL-based Photonic System Architecture over a Multi-hop Path with 19-Core MCF for Future Agile Tb/s Metro Networks
}

\author{
M. Svaluto Moreolo ${ }^{1}$, J. M. Fabrega ${ }^{1}$, L. Nadal ${ }^{1}$, R. Martínez ${ }^{1}$, R. Casellas ${ }^{1}$, J. Vílchez ${ }^{1}$, R. Muñoz ${ }^{1}$, R. \\ Vilalta $^{1}$, A. Gatto ${ }^{2}$, P. Parolari ${ }^{2}$, P. Boffi ${ }^{2}$, C. Neumeyr ${ }^{3}$, D. Larrabeiti ${ }^{4}$, G. Otero ${ }^{4}$, J. P. Fernández-Palacios ${ }^{5}$ \\ ${ }^{1}$ Centre Tecnologic de Telecomunicacions de Catalunya (CTTC/CERCA), Castelldefels (Barcelona), Spain; ${ }^{2}$ Politecnico di Milano, Milan, Italy; \\ ${ }^{3}$ Vertilas GmbH, Garching, Germany; ${ }^{4}$ Universidad Carlos III de Madrid, Spain; ${ }^{5}$ Telefonica Global CTO, Madrid, Spain \\ michela.svaluto@cttc.es
}

\begin{abstract}
An SDN-enabled photonic system adopting VCSEL technology is experimentally analyzed targeting dynamic 5G-supportive MAN. Direct and coherent detection modules are compared and programmability assessed over up to 6-hop 160km HL4-HL2/1 connection including 25km 19-core MCF. @ 2020 The Author(s)
\end{abstract}

\section{Introduction}

The Metropolitan Area Network (MAN) is characterized by stringent requirements in terms of traffic volume [1], traffic variance, cost and power efficiency. In order to achieve a cost-effective ultra-broadband transport featuring dynamic capacity adaptation, novel architectures must be envisioned seeking for programmability and efficient photonic technologies [2]. This way the MAN will be able to deal with the challenges posed by the ever-increasing access rate offers, the support of $5 \mathrm{G}$ new radio backhaul/fronthaul traffic or the scalable provision of $\mathrm{Gb} / \mathrm{s}$ mobile service in events gathering crowds of people. Finally, also CDN and corporative VPN traffic will continue to be large traffic flows traversing the MAN in the next years that need support [1].

In the framework of EU-H2020 PASSION project, we propose to approach the MAN challenges by adopting vertical cavity surface emitting laser (VCSEL) technology at long wavelengths with very high bandwidth ( $\geq 18 \mathrm{GHz})$ and dense photonic integration, to design programmable photonic sliceable bandwidth/bitrate variable transceivers (S-BVTs) with reduced cost, power consumption and footprint [2]. They can be modularly composed following a pay-as-you-grow scheme with license-based module activation, fully exploiting the available resources. Fine granularity is enabled by direct modulation (DM) of VCSEL with multicarrier technique. The programmability and automation of photonics is key to support hyperconnectivity [2]. Thus, we propose using a software defined networking (SDN) platform to enable a flexible, efficient and dynamic exploitation of the optical layer through smart traffic aggregation, statistical multiplexing and IP offloading at the MAN transit nodes.

In this paper, we analyze the performance of the proposed architecture up to 6 aggregation nodes and $160 \mathrm{~km}$ (see Fig. 1), as a worst case path across multiple hierarchical levels (HL) in a 5G-supportive MAN based on real topologies [3, 4]. Different HL node functionalities are considered according to the specific architecture, as described in [3]. Lower level metro-aggregation nodes (HL4, aggregating traffic from edge nodes at HL5) are equipped with simple and cost-effective VCSEL-based transceiver (up to 2Tb/s) and switching technology exploiting spectral dimension. Higher level metro-core nodes (HL2/1) adopt fully-equipped (with modular approach) transceivers (up to $16 \mathrm{~Tb} / \mathrm{s}$ ) and photonic switching nodes supporting multiple functionalities and granularities, handling both spectral and spatial dimensions. To experimentally assess the performance of the photonic system architecture, a single flow connection enabled by a specific VCSEL is considered over up to 6 hops and $160 \mathrm{~km}$, including a real testbed network (ADRENALINE) and $25 \mathrm{~km} \mathrm{19-core} \mathrm{multicore} \mathrm{fiber} \mathrm{(MCF).} \mathrm{We}$ analyze both direct detection (DD) and coherent receiver (CO-Rx) options, showing that the latter achieves promising results for multi-hop MAN connections. Furthermore, the programmability via software defined networking (SDN) to configure S-BVTs and nodes is also verified.

\section{SDN-enabled modular photonic system architecture and experimental set-up}

Fig. 1 shows the SDN-enabled photonic modular system architecture based on the EU-H2020 PASSION approach [3]. The fundamental S-BVT module consists of 40 DM-VCSELs (4 submodules with 10 VCSELs each), integrated on a silicon-on-insulator (SOI) chip with $100 \mathrm{GHz}$-spaced operating wavelengths within the C-band, supporting up to $2 \mathrm{~Tb} / \mathrm{s}(50 \mathrm{~Gb} / \mathrm{s}$ per each independent flow). To fully exploit the spectral resource, 4 modules generating $25 \mathrm{GHz}-$ spaced flows are required to obtain a fully-equipped (super module) S-BVT transmitter (Tx) supporting up to 8Tb/s (inset Fig. 1). This capacity can be doubled exploiting the polarization division multiplexing (PDM) and improve by a factor $M$, considering also space division multiplexing (SDM) with a MCF with $M$ cores. Photonic integrated 
circuit (PIC) CO-Rx modules are envisioned to improve the capacity/reach performance. In the target 5G-supportive MAN, based on real topologies, the most challenging goal is featuring very high capacity (about $1 \mathrm{~Tb} / \mathrm{s}$ ) multi-hop all-optical circuits from HL4 to HL2/1 over a path length that in the worst case can reach about 150km [4]. HL4 nodes are equipped with simpler S-BVT (e.g. the fundamental module) and enable simple add/drop functionality, adopting 100/50GHz multiplexers/demultiplexers and wavelength blockers. The transit nodes at HL3 have the same advanced functionalities as HL2/1 nodes, which are equipped with full-featured high-capacity S-BVTs (Fig. 1 insets). The add/drop and aggregation/disaggregation nodes are based on wavelength selective switch (WSS) to handle finer granularities; while a photonic switch (PS) module is included to handle the spatial dimension. S-BVTs and nodes are programmable by an SDN controller via specific SDN agents (as shown in Fig. 1).

For the experimental assessment, a single flow of the S-BVT is enabled. At the S-BVT Tx, adaptive digital signal processing (DSP) based on orthogonal frequency division multiplexing (OFDM) with bit loading (BL) algorithm and a 64GSa/s digital to analog converter (DAC) are used for direct modulating a $10 \mathrm{GHz}$ bandwidth single-mode VCSEL. It operates at $1545.32 \mathrm{~nm}(194.00 \mathrm{THz})$, which corresponds to a nominal central frequency $(n)$ of 144 according to [5] for flexi-grid and represents VCSEL number 6, within submodule 2 of SOI-chip fundamental module 1 (Fig.1 left inset). For the S-BVT Rx, we consider both DD and CO-Rx module options. The former is a PIN diode with $20 \mathrm{GHz}$ bandwidth and includes a transimpedance amplifier (TIA); the latter has discrete components (up to $40 \mathrm{GHz}$ bandwidth) and includes a tunable local oscillator (LO). The received signal is captured by an oscilloscope (at $50 \mathrm{GSa} / \mathrm{s}$ ) and post-processed by adaptive DSP. Based on the worst case analysis of the path from HL4 to HL2/1, for the experimental set-up we consider a 6-hop 160km path traversing up to 6 WSSs and two $100-\mathrm{GHz}$ arrayed waveguide gratings (AWGs), including a real testbed network (ADRENALINE) and 25km MCF with fan-in and fan-out. The WSS at the Tx is for single sideband (SSB) filtering. The AWGs adopted at the first node (N1) forward traffic from the S-BVT towards the upper HL. According to the envisioned HL3 and HL2/1 node architecture [3], the WSS-based filtering stages at N2 and N4 nodes implement the add functionality, while at N3 node an additional WSS is included to take into account the aggregate/disaggregate functionality to spectrally combine the considered flow with other flows at the HL3 node. In addition to the PSs at the ADRENALINE nodes (N2, N3 and N4 in Fig. 1), variable and fixed attenuators have been considered in the set-up in order to emulate the PS modules present at HL3 and HL2/1 nodes [3]. Particularly, a 2-dB attenuator (Att.) after the 19-core MCF emulates the pass-through functionality at N5 node. In the last HL2/1 node (N6), a WSS is considered for dropping the traffic towards the S-BVT Rx. The node WSS bandwidth has been set to 50GHz. As shown in Fig. 1, the total path length is $160 \mathrm{~km}$. The $35 \mathrm{~km}$ standard single-mode fiber (SSMF) links within the ADRENALINE network are pre-amplified by means of EDFAs, in the rest of the set-up optical amplifiers (OAs) are indicated.

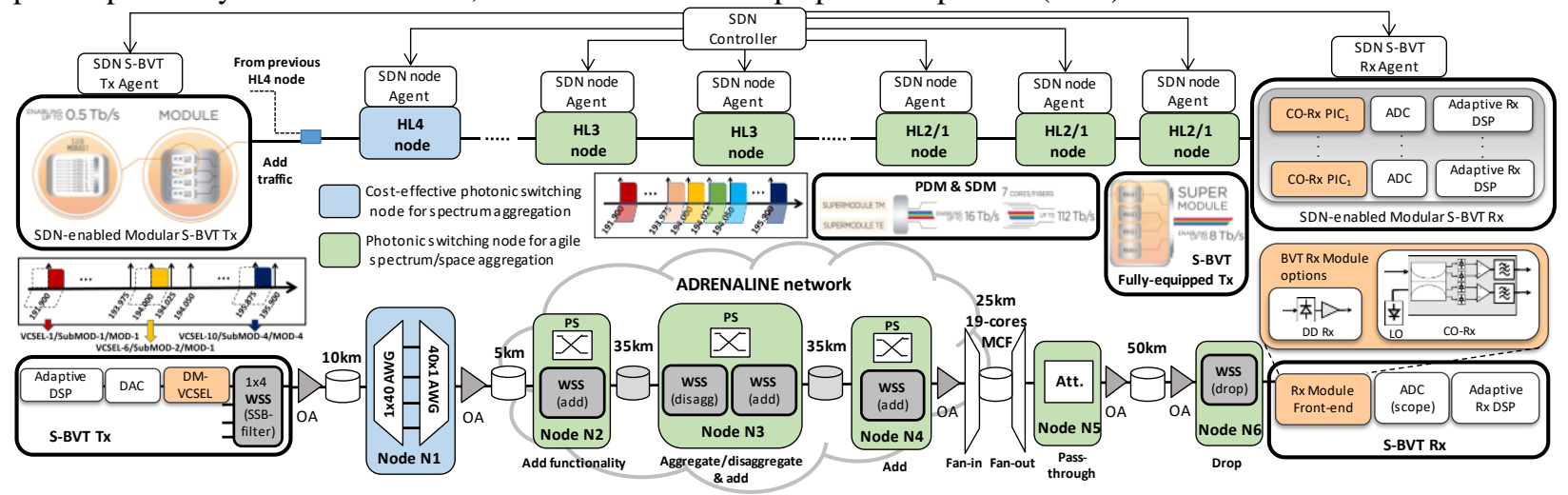

Fig. 1. Schematic of SDN-enabled modular photonic system architecture for a HL4-HL2/1 connection (top); experimental set-up (bottom).

\section{Performance assessment and programmability}

We first analyze the back-to-back (B2B) performance of the S-BVT based on the 10GHz VCSEL adopting either DD or CO-Rx. At the offline DSP, an OFDM signal, with 512 subcarriers over $16 \mathrm{GHz}$, is generated. Uniform loading (UL) is used to estimate the signal to noise ratio (SNR) and BL is applied for rate adaptive algorithm; the target BER is set to $4.62 \cdot 10^{-3}$, considering HD-FEC with $7 \%$ overhead for successful transmission [2]. Fig. 2a shows the results at the varying of optical SNR (OSNR). The maximum capacity achieved with DD is $36.9 \mathrm{~Gb} / \mathrm{s}$, while with $\mathrm{CO}-\mathrm{Rx}$ is $31.2 \mathrm{~Gb} / \mathrm{s}$. This is due to the adopted CO-Rx, which is implemented with discrete components. In fact, by adopting a commercial integrated CO-Rx with similar responsivity, a capacity of $34.6 \mathrm{~Gb} / \mathrm{s}$ (at $33.4 \mathrm{~dB}$ OSNR) is supported, outperforming not only the CO-Rx used in this experiment, but also the DD for the same OSNR (Fig. 2a). Nevertheless, even the discrete CO-Rx presents better performance than DD for values of OSNR lower than $26.7 \mathrm{~dB}$ 
$(23.7 \mathrm{~Gb} / \mathrm{s})$. After the characterization of the Rx modules, we analyze a HL4-HL3 connection (including N1 and N6) over $15 \mathrm{~km} 2$-hop path. In this case, the OSNR is $35.7 \mathrm{~dB}$ and the capacity of DD $(33.7 \mathrm{~Gb} / \mathrm{s})$ and CO-Rx $(26.4 \mathrm{~Gb} / \mathrm{s})$ are similarly affected (slight decrease compared to B2B). Finally, we analyze the HL4-HL2/1 connection over the 160km 6-hop path, including the testbed network and the MCF (as in Fig. 1). The OSNR decreases to 26.4dB; the CO-Rx supports $22.6 \mathrm{~Gb} / \mathrm{s}$, while the capacity with DD is lower than $10 \mathrm{~Gb} / \mathrm{s}$ (Fig. 2a). In fact, even if a simplified CO-Rx DSP is adopted due to intensity modulation at Tx [2], improved performance can be obtained after $160 \mathrm{~km}$ thanks to a compensation module (CM) at CO-Rx DSP (Fig. 2b). Thus, the B2B capacity with CO-Rx decreases about $15 \%$ and $28 \%$ over HL4-HL3 and HL4-HL2/1 connections, respectively. Adopting higher bandwidth VCSEL and integrated CO-Rx, it is envisioned to improve the achievable capacity/reach to target up to 50G/s per flow [3].

The configuration of the different network elements (i.e., S-BVT Tx/Rx and nodes) is coordinated by an SDN controller. A defined REST API [6] enables the communication between the SDN controller and every network element agent. Fig. 2c depicts the set of REST API messages for: i) allocating the S-BVT Tx element (i.e., VCSEL) at $194.000 \mathrm{THz}(n=144)$ (see JSON encoding in Fig.2e); ii) configuring the S-BVT-Rx LO at $194.000 \mathrm{THz}(n=144)$; iii) programming the cross-connections at each traversed HL node with its frequency slot (FS) in terms of $n$ and slot width as $2 \cdot m \cdot 6.25 \mathrm{GHz}$. Fig. $2 \mathrm{~d}$ shows a protocol analyzer screenshot with the REST API messages validating the workflow. Since different HL nodes present different filtering capabilities (HL4 uses $100 \mathrm{GHz}$ whilst HL3 and HL2/1 use 50GHz), for each HL node cross-connection, in addition to specifying the input and output ports, the FS needs to be determined, according to the filtering switch features. For HL4 nodes, the FS has $m=8$ (i.e. slot with of $100 \mathrm{GHz}$ ), whereas for both HL3 and HL2/1, $m=4$ resulting in a slot width of $50 \mathrm{GHz}$ (see Fig. 2f).

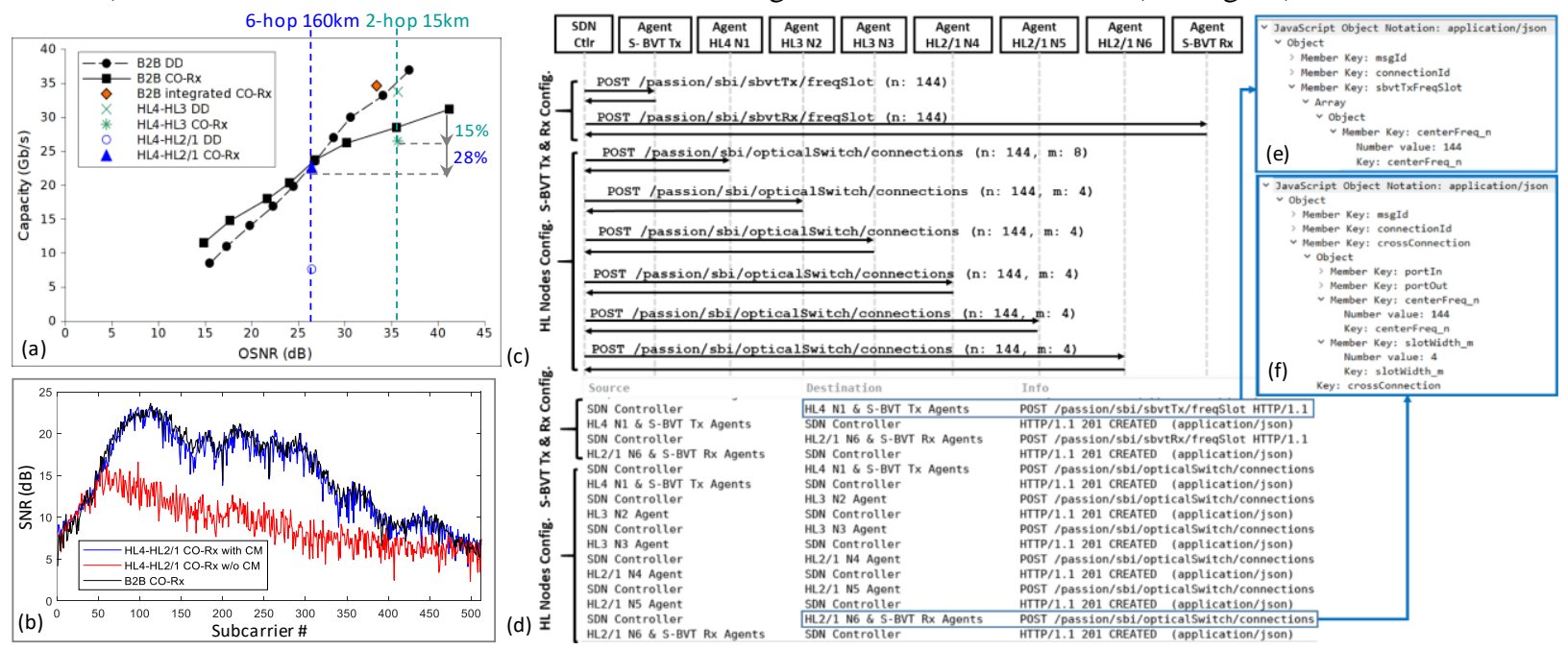

Fig. 2. (a) Capacity vs OSNR for different Rx modules and connections; (b) SNR vs subcarrier number. (c) Workflow for configuring SBVT Tx/Rx at source/destination nodes along with FS over every traversed HL node; (d) validation of REST API messages enabling S-BVT and HL nodes programmability; (e) JSON with S-BVT Tx configuration (n: 144); (f) JSON with HL2/1 N6 programmability (n: 144, m: 8).

\section{Conclusions}

We have assessed an SDN-enabled photonic system architecture, targeting 5G-supportive MAN, across multiple HL, enabling IP-offloading through up to 6 aggregation nodes and $160 \mathrm{~km}$, including a real testbed network and MCF. DD and CO-Rx are compared, showing that while DD is more affected by OSNR degradation and path length increase, with 10GHz VCSEL, CO-Rx allows supporting >30Gb/s in B2B and >20Gb/s capacity over the multi-hop path. Adopting programmable (SDN-enabled) photonic transceivers based on $>18 \mathrm{GHz}$ VCSELs and integrated CO$\mathrm{Rx}$, exploiting spectral and spatial dimensions, the proposed solution is promising to target Tb/s MAN connections. Work funded by the EU H2020 PASSION project GA 780326 and the Spanish AURORAS project (RTI2018-099178-B-I00).

\section{References}

[1] Cisco Visual Networking Index: Forecast and Trends, 2017-2022, Nov 2018.

[2] M. Svaluto Moreolo, et al., "Synergy of Photonic Technologies and Software-Defined Networking in the Hyperconnectivity Era," IEEE/OSA Journal of Lightwave Technology, 37, 16, $3902-3910$ (2019).

[3] M. Svaluto Moreolo, et al., " Spectrum/Space Switching and Multi-Terabit Transmission in Agile Optical Metro Networks," in Proc. OECC/PSC, Fukuoka, Japan, July 2019.

[4] D. Larrabeiti, et al."All-Optical Paths across Multiple Hierarchical Levels in Large Metropolitan Area Networks," in Proc. ACP, Nov. 2019.

[5] ITU-T G694.1 Rec., "Spectral grids for WDM applications: DWDM frequency grid", Feb., 2012.

[6] R. Martínez, et al., "Proof-of-Concept Validation of SDN-Controlled VCSEL-based S-BVTs in Flexi-Grid Optical Networks," in Proc. OFC 2019, San Diego, USA, March 2019. 